\title{
Effect of live Salmonella Ty21a in Dextran Sulfate Sodium-induced Colitis
}

\author{
Gunnar Nysœter ${ }^{1}$, Kari Erichsen², Anne Marita Milde ${ }^{3}$, Eva Colás ${ }^{4}$, Einar \\ Kristoffersen ${ }^{5}$ and Arnold Berstad ${ }^{6}$ \\ ${ }^{1}$ Department of Medicine, Section for Gastroenterology, ${ }^{2}$ Children's Clinic, ${ }^{3}$ Department of Biological \\ and Medical Psychology, ${ }^{4}$ Innovest, ${ }^{5}$ Section for Microbiology and Immunology, Haukeland University \\ Hospital, Bergen, Norway, ${ }^{6}$ Institute of Medicine, University of Bergen, Norway.
}

\section{Abstract}

Background: Intestinal microbiota seems to play an essential role in the development of inflammatory bowel diseases (IBD). We hypothesised that an oral vaccine based on live Salmonella typhi would be well tolerated and could even attenuate dextran sulfate sodium (DSS) induced colitis in rats, an animal model of IBD.

Methods: Nine male Wistar rats was used for an initial tolerance study, in which we used 3 dose-levels of Salmonella Ty21a, $0.5 \times 10^{9}, 1 \times 10^{9}$, and $2 \times 10^{9} \mathrm{CFU}$, each dose being tested in 3 rats. Four treatment groups consisting of 8 male Wistar rats per group: 1) control group given standard food and water, 2) control group given four daily administrations of Salmonella Ty21a $1 \times 10^{9} \mathrm{CFU}, 3$ ) water with $5 \%$ DSS the last 7 days, 4) four daily administrations of Salmonella Ty21a before water with 5\% DSS the last 7 days. The Salmonella Ty21a was administered by gastric gavage on day 1,3, 5 and 16, while DSS was given with the drinking water from day 15 to 22. The animals were sacrificed and colonic tissue removed for analysis 22 days after gavage of the first vaccine dose.

Results: The animals in the tolerance study got no signs of disease. In the treatment study, all animals receiving DSS had histologic indications of colitis, particularly in the distal part of the colon. Administration of Salmonella Ty21a had no significant effect on crypt and inflammation scores $(\mathrm{p}>0.05)$.

Conclusion: Gastric administration of live vaccine strain Salmonella Ty21a was well tolerated, but did not provide any significant protection against development of DSS induced colitis in rats.

Keywords: salmonella Ty21a, colitis, rats, inflammatory bowel disease

\section{Introduction}

About $0.2 \%$ of the population in Scandinavia suffer from ulcerative colitis or Crohn's disease, collectively called chronic inflammatory bowel disease (IBD) (Fonager, Sorensen and Olsen, 1997; Lapidus 2006; Moum et al. 1996). But while the prevalence and cost of the disease is increasing, to find the aetiology and causal treatment remain a huge challenge. Traditionally, IBD is considered an autoimmune disease, and treatment with immunosuppressive drugs has had a prominent position. More recently, IBD has been ascribed to an inadequate mucosal immune response to the intestinal microbiota in genetically susceptible individuals. Consequently it might be better to stimulate the immune defence of the intestine rather than suppressing a secondary inflammatory response. There is in fact mounting evidence that immune stimulation of the intestinal epithelium is one way to treat inflammatory conditions in the gut. Thus, a study with granulocyte - macrophage colony-stimulating factor (GM-CSF), a myeloid growth factor, has shown promising results in Crohn's disease (Korzenik et al. 2005).

Several animal studies on experimental colitis have shown a protective or therapeutic effect from probiotics or vaccines (Boirivant et al. 2001; Fujiwara et al. 2003; Madsen et al. 2001; Ohman 2005; Osman et al. 2004). A similar effect on humans with IBD has presently not been shown, except possibly for probiotics (Bibiloni et al. 2005; Kanauchi et al. 2003). The vaccine strain Salmonella Ty21a is of interest in this connection. Containing live bacilli it may theoretically combine the effects of probiotics and vaccines used in other studies. Extensive clinical use has proved that the vaccine is

\section{Correspondence:}

Copyright in this article, its metadata, and any supplementary data is held by its author or authors. It is published under the Creative Commons Attribution By licence. For further information go to: http://creativecommons.org/licenses/by/3.0/. 
remarkably well tolerated, even by IBD-patients with moderate disease activity (Engels et al. 1998; Sands et al. 2004). One may speculate whether salmonella infections have some relation to IBD, considering the inverse prevalence of the two diseases. We even have reports from a few of our IBD-patients that the course of their IBD has become milder after they have been vaccinated against typhoid fever with this live vaccine. This has been a surprising observation as they were vaccinated before travelling to endemic areas and did not anticipate any effect on their chronic disease.

Results from animal studies have limited value for the understanding of IBD. But as a first step we wanted to see if experimental colitis could be influenced by live salmonella vaccine in a similar way that was previously found with probiotics and some other vaccines. We therefore studied whether administration of the live vaccine strain, Salmonella Ty21a would influence the induction of colitis by dextran sulphate sodium (DSS) in rats, a well-known model of ulcerative colitis (Kim and Berstad, 1992).

\section{Materials and Methods}

\section{Animals and husbandry}

Forty-one male Wistar rats (Taconics Europe, Skensved, Denmark), 6 weeks old with mean weight of $147 \mathrm{~g}$ (95\% CI 143.5-149.9), were housed individually in Makrolon III cages in an open system. They were kept under standard laboratory conditions with a temperature of $21 \pm 1{ }^{\circ} \mathrm{C}$, dark/light cycles of $12 / 12$ hours, relative humidity of $55 \% \pm 5 \%$, and 20 air changes per hour. Access to food, SDS RM1 (E) (Scanbur BK AS, Nittedal, Norway) was ad libitum. Tap water was given ad libitum if not otherwise stated. The Norwegian Animal Research Authority approved the protocol.

\section{Induction of colitis}

Acute colitis was induced by $50 \mathrm{~g} / \mathrm{L}$ of DSS (MW 44000; TdB Consultancy AB, Uppsala, Sweden) given in distilled drinking water for 7 days.

\section{Test substance}

Salmonella serovar Ty21a (Vivotif ${ }^{\circledR}$; Berna, Como Italy), $2 \times 10^{9}$ colony forming units (CFU) freeze dried live bacilli per capsule. The content of one capsule was dissolved in $2 \mathrm{~mL} \mathrm{NaCl} 0.9 \%$ immediately before use.

\section{Antibody response}

Serum salmonella antibodies were measured semiquantitatively by bacteria agglutination using the Widal reaction with O-antigens (SIFIN Gmbh, Berlin, Germany).

\section{Experimental Protocol}

After 7 days of acclimatisation, 9 animals were divided at random into 3 groups of 3 animals for the dose-tolerance study, while the remaining 32 animals waited for the colitis study starting one week later.

\section{Dose-tolerance study}

The 9 animals were given Ty21a; one group with $0.5 \times 10^{9}$, one with $1 \times 10^{9}$, and one with $2 \times 10^{9}$ CFU. Each dose was given three times at two days interval. Six days after the first dose the animals were sacrificed and blood culture was taken. We registered general condition, consistency and blood content of the stool daily.

\section{Treatment study}

The animals were divided into the following four groups of 8 rats in each group: (1) Control. (2) Ty21a alone. (3) DSS alone. (4) DSS + Ty21a. Rats in the groups 2 and 4 got $1 \times 10^{9}$ CFU Salmonella Ty $21 \mathrm{a}$ in $1 \mathrm{~mL}$ saline $0.9 \%$ through a metallic oral tube on day 1, 3, 5 and 16. In group 3 and 4 drinking water was replaced by DSS 5\% in distilled water from day 15 to 21 . Group 1 remained on normal food and drinking water during the whole test period. The rats were weighed at start of treatment with Ty21a (day 1), after 7 and 14 days, and then daily until sacrifice (day 22). On day 22 the animals were anaesthetised by subcutaneous injection of a combination of phenantyl citrate + fluanisone (Hypnorm; Jansen Pharmaceutica, Beerse, Belgium) and midazolam (Dormicum; Roche, Oslo, Norway). (Phenantyl citrate $0.079 \mathrm{mg} / \mathrm{mL}$, fluanisone 2.5 $\mathrm{mg} / \mathrm{mL}$, midazolam $1.25 \mathrm{mg} / \mathrm{mL}$ given at dose $0.2 \mathrm{~mL} / 100 \mathrm{~g}$.)

Thoracotomy, cardiac puncture and exsanguination were performed. The colon was taken out. 


\section{Sample collection and analyses}

Stool was observed daily from day 15 to 21 for the presence of diarrhoea or blood. We used a guyac method (Haemofec. Med-Kjemi A/S, Asker, Norway).

Granulocyte marker protein (GMP), the rat equivalent to human calprotectin, was measured in stool (Kristinson et al. 2002; Milde et al. 2003). Samples for analysis were collected on day 1, 7 and 21. They were stored at $-20{ }^{\circ} \mathrm{C}$ until analysis for GMP. On exsanguination blood was drawn for blood culture and for analysis of salmonella antibodies.

\section{Tissue preparation and histologic examination}

Colon from the colocecal junction to the anal verge was removed. The length of the colon was recorded. The colon was rinsed with phosphate-buffered saline, opened longitudinally, and divided into one proximal and one distal segment, which were fixed in $10 \%$ formalin and embedded in paraffin. Eight pieces per segment were stained with hematoxylin and eosin and specimens coded and randomised before microscopic examination.

Crypt and inflammatory scores were determined according to a validated scoring system (Carrier et al. 2001). Crypt injury was scored as follows: grade 0 , intact crypts; grade 1, loss of the bottom third of crypts; grade 2, loss of the bottom two thirds of crypts; grade 3, loss of entire crypt with the surface epithelium remaining intact; grade 4 , loss of entire crypt and surface epithelium. The severity of inflammation was scored as follows: grade 0 , normal; grade 1 , focal inflammatory cell infiltration; grade 2 , inflammatory cell infiltration, gland drop out, and crypt abscess. Both scores include a measure of involvement as follows: grade1, $1 \%$ to $25 \%$; grade $2,26 \%$ to $50 \%$; grade $3,51 \%$ to $75 \%$; grade $4,76 \%$ to $100 \%$. The score was the product of either the crypt or inflammation grade by the involvement grade.

\section{Statistical analysis}

Data were analysed using the GraphPad Prism version 4 (GraphPad Software, San Diego, Calif.) statistical software package. Results are presented as mean \pm SEM. Differences between means were evaluated with 1-way Anova and Bonferroni posttest for selected pairs of columns.

Differences between means and 95\% confidence intervals (CIs) are given if not otherwise stated. $\mathrm{p}$ values less than 0.05 were considered statistically significant.

\section{Results}

\section{Tolerance study}

All the animals were well during the test period. None got diarrhoea or bloody stools. Blood cultures were all negative.

\section{Treatment study}

All the animals appeared to be at good condition until sacrifice.

\section{DSS Intake}

Total intake of DSS-containing water was similar in the DSS alone $(161.0 \pm 8.1 \mathrm{~mL})$ and the DSS + Ty21a groups $(168 \pm 8.4 \mathrm{~mL} ; \mathrm{p}=0.45)$. There was no significant difference of drinking volume in the four groups.

\section{Weight change}

All rats gained weight without significant differences between groups until DSS treatment was started on day 15. During the following period until day 22 both groups on DSS had significantly less weight gain than the animals in the control or Ty21a-groups. (Fig. 1) There was no significant difference in weight change between the DSS group $(4.75 \pm 2.15)$ and the DSS + Ty21a group $(-0.75 \pm 2.82 ; \mathrm{p}=0.25)$.

\section{Stool changes}

None of the animals got diarrhoea. Blood in stools was found in six animals in both the DSS group and those on DSS + Ty21a, four with occult blood and two with traces of visible blood in each group.

\section{Colon length}

Colon length did not differ significantly between the groups, though there was a tendency to shorter colon in the groups that got DSS $(14.13 \pm 0.42 \mathrm{~cm})$ or DSS + Ty21a (13.69 $\pm 0.49 \mathrm{~cm} ; \mathrm{p}=0.35)$.

\section{Macroscopic lesions of the gut}

Intramucosal bleeding or erosions was observed in the colon of a few animals both in the DSS group (4 animals) and the DSS + Ty21a group (5 animals), 


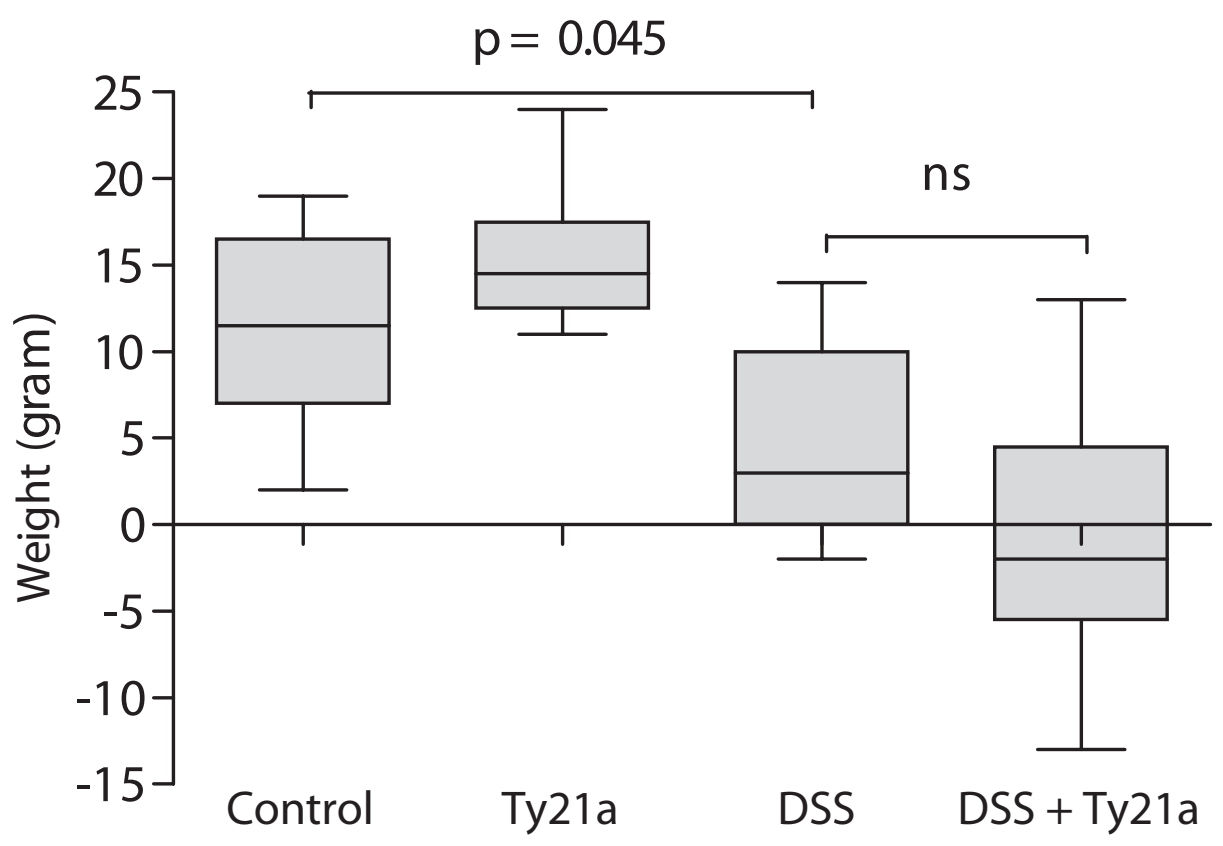

Figure 1. Weight gain from day 14-22 (DSS-period).

while in the other groups, the colons were macroscopically normal.

\section{Granulocyte marker protein}

Compared with controls, DSS-induced colitis significantly increased faecal GMP by $100 \mathrm{mg} / \mathrm{L}(95 \%$ CI 118-81; $p<0.0001)$. There was no significant difference of GMP-levels in animals on DSS (105 \pm 8) or DSS + Ty21a (108 $\pm 8 ; \mathrm{p}=0.5)$. (Fig. 2).

\section{Salmonella antibodies}

Salmonella antibodies were found in 7 of the 8 animals that got Ty21a and in all of the eight animals that got DSS + Ty21a. The other animals had no salmonella antibodies. There was no correlation between antibody levels and tissue damage.

\section{Blood culture}

There was growth of Gram positive cocci, not salmonella, in one sample from an animal in the control group, no growth in culture from the others. As no rats showed signs of septicaemia, the positive blood culture most likely reflects contamination upon blood sampling.

\section{Histology}

Histologic findings were normal or close to normal in all animals in the control group and the Ty21a group, while all animals on DSS, with or without the addition of Ty21a developed inflammatory changes in the colon (Fig. 3). The mean value of crypt score was slightly less for the DSS + Ty21a group compared to the DSS group (Fig. 4), but the difference was not statistically significant $(95 \% \mathrm{CI}$ of diff. -0.63 to $1.55 ; \mathrm{p}>0.05$ ). Analysis of inflammatory score showed no significant differences between the two groups ( $95 \%$ CI of diff. -0.63 to 0.73; $p>0.05$ ), (Fig. 4). There was a significant correlation between GMP values and histologic findings (Pearson's $\mathrm{r}=0.77$; $\mathrm{p}<0.0001$ ).

\section{Discussion}

We failed to find a protective effect of salmonella Ty21a in DSS-induced colitis. Both histological signs of colitis and GMP levels in blood indicated similar degree of inflammation in DSS and DSS + Ty21a treated groups. The two methods support each other as they both depend on the amount of granulocytes infiltrating the intestinal mucosa. Mucosal damage as measured by crypt score was numerically less pronounced in vaccinated animals, but the difference was not statistically significant. However, neither the initial tolerance study nor the controlled study indicated any adverse effects of the treatment. Although the results should be carefully extrapolated, the finding of no deterioration of the colitis, suggests that 


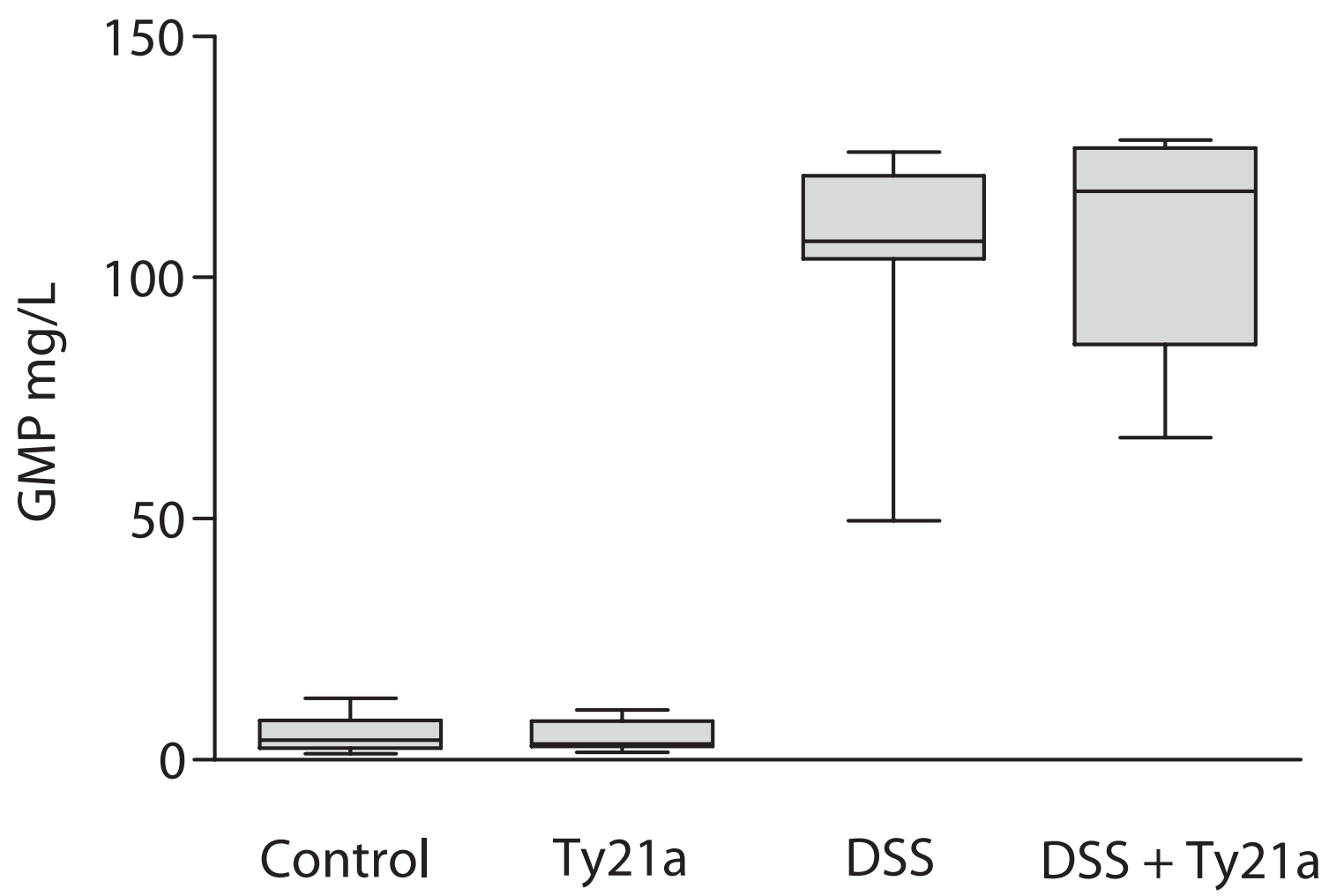

Figure 2. Salmonella Ty21a does not affect the level of granulocyte marker protein (GMP) in rats.
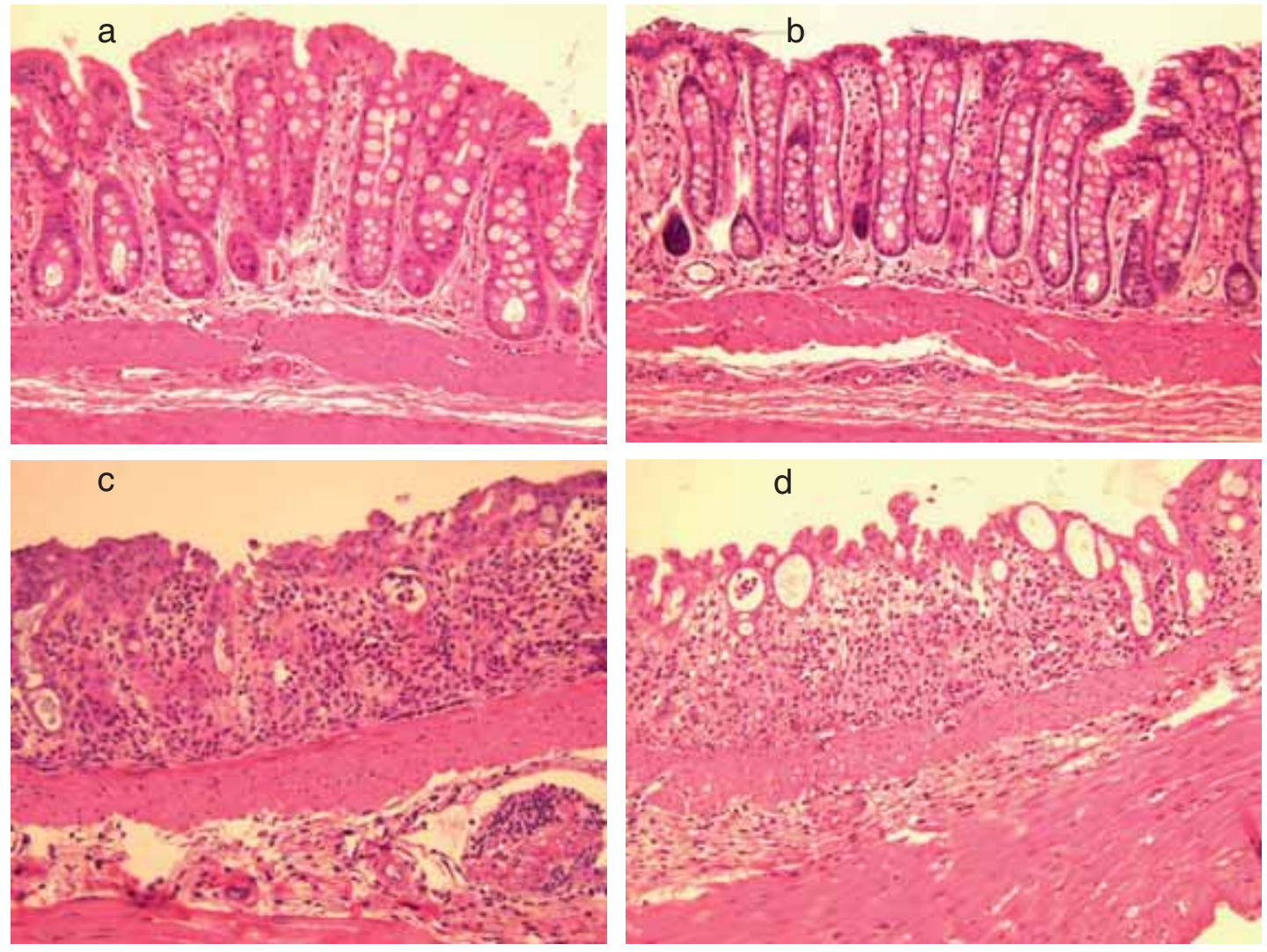

Figure 3. H\&E $\times 20$. Histologic normal mucosa in healthy control rat (a) and healthy Ty21a rat (b). Mucosa from DSS-group (c) and DSSTy21a-group (d) with severe inflammatory changes with crypt loss, crypt abscess and infiltration with inflammatory cells. 

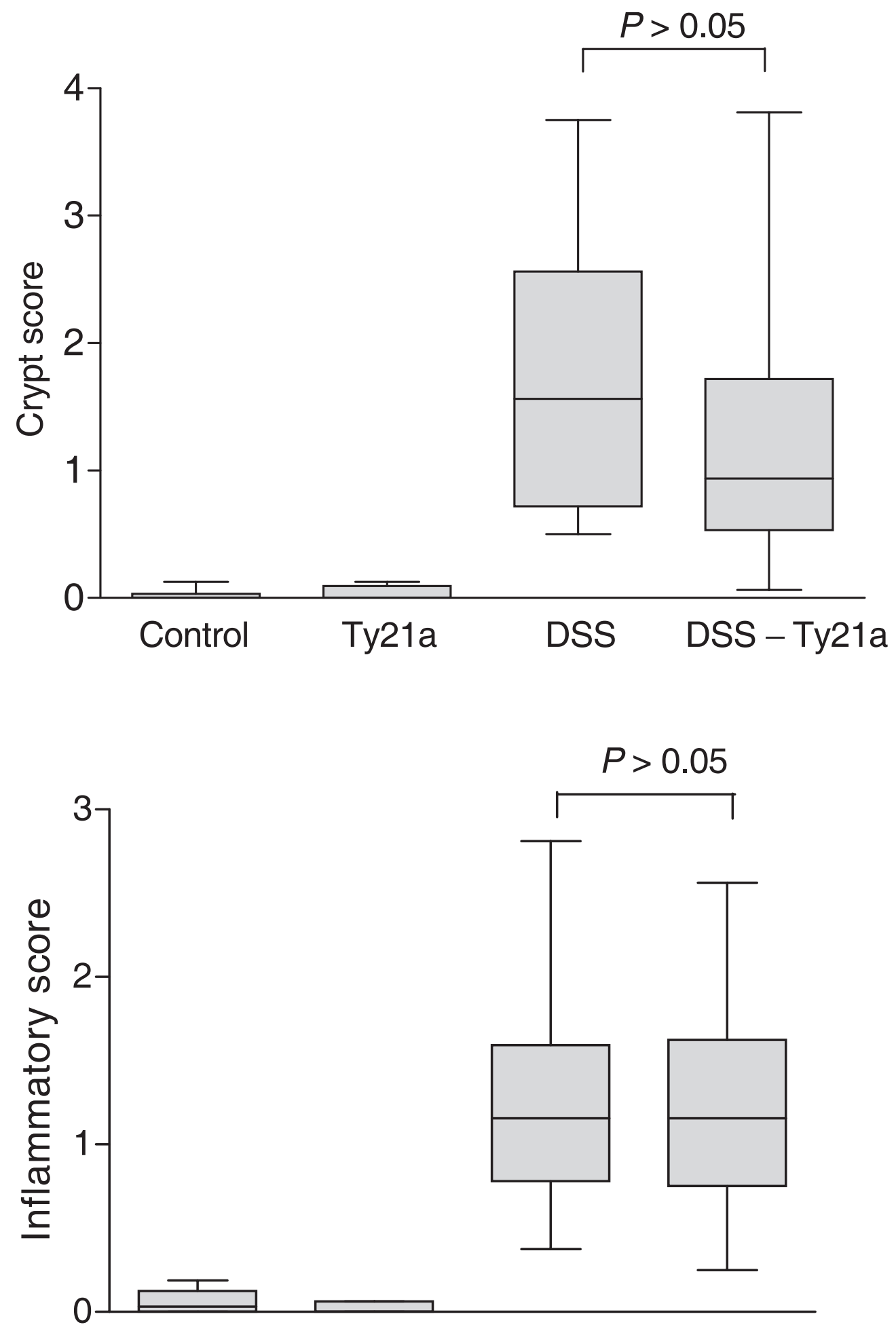

Control Ty21a DSS DSS + Ty21a

Figure 4. Histologic findings (crypt score and inflammatory score) in dextran sulphate induced colitis in rats is not significantly influenced by treatment with Salmonella Ty21a. 
vaccination with Salmonella Ty21a in humans with IBD is safe. This corresponds well with extensive experience in humans. Current guidelines for vaccination of patients with IBD allow oral administration of Salmonella Ty21a provided the patient is not significantly malnourished or immune compromised (Sands, Cuffari, Katz, Kugathasan, Onken, Vitek and Orenstein, 2004). Any information as to whether the vaccine influences the IBD itself is not available.

For human use salmonella Ty21a is taken in the encapsulated form to avoid destruction of the microbes by pepsin and acid in the stomach. Our rats got unprotected vaccine by gavage through a metallic sonde, assuming that a sufficient proportion of the high dose of live microbes (half that given to an adult person) would reach the gut. The three first doses of vaccine were given on day 1,3 and 5 as is the recommended schedule for human use. The following two weeks interval enabled the animals to face the DSS-challenge from day 15 with an immune system triggered by Ty21a. All the vaccinated rats, except one, produced salmonella antibodies, thus proving the vaccine's contact with the immune system. To achieve a possible probiotic effect, a higher vaccine dose could be necessary. But instead we chose to give a fourth dose the day after DSS-treatment was started (day 16), in order to have live salmonella present in the gut during the colitis period. The choice of timing for vaccination as well as duration of the colitis period may be essential for the effect ( $\mathrm{Di}$ Giacinto et al. 2005; Jun et al. 2005). To have a better parallel to a clinical situation, we might have induced a chronic colitis first, and then introduced the vaccine to study its therapeutic effect. All animals receiving DSS got colitis, particularly in the distal part of the colon, but the inflammation was remarkably weak compared with what we observed in a recent study performed by us (Erichsen et al. 2005; Milde and Murison, 2002). The relevance of this fact, is not known.

A vaccine containing live bacilli could theoretically have the advantages of both probiotic and vaccine components. Accordingly we gave the vaccine both two weeks before and during the induction of colitis. As assumed, the adaptive immune system had started production of salmonella antibodies after two weeks. Others have found that in humans, oral vaccination with live Salmonella Ty21a stimulated, during the first few weeks, large amounts of IFN- $\gamma$ but no IL-10 or
TGF- $\beta$ (Lundin, Johansson, and Svennerholm, 2002), which theoretically could be an unfavourable effect of Salmonella Ty21a in IBD. However, the activity of such pro-inflammatory Th1 cytokines may be subdued by subsequent induction of $\mathrm{CD} 4{ }^{+} \mathrm{CD} 25^{+}$regulatory $\mathrm{T}$ lymphocytes producing both IL-10 and TGF $\beta$ with a healing effect on colitis (Holmen et al. 2006; Uhlig et al. 2006). In case of the latter, a study with a longer observation period would be of interest. A live vaccine against enterotoxigenic Eschericia coli (ETEC) suppressed the proinflammatory response (Jun, Gilmore, Callis, Rynda, Haddad and Pascual, 2005) and reduced the production of TNF- $\alpha$, IL-1 and IL- 6 , while it increased the production of IL-4, IL-10, and IL-13. Interestingly, toxins associated with another tropic diarrhoeal disease, cholera, has an anti-inflammatory effect in experimental TNBS-colitis. Oral administration of recombinant cholera toxin subunit B has been shown to inhibit murine TNBSinduced experimental colitis (Boirivant, Fuss, Ferroni, De Pascale and Strober, 2001) and promote a Th2 response with $\operatorname{Tr} 1$ cells, enhanced IL-10 production and inhibition of IL-12 and TNF$\alpha$ secretion (Lavelle et al. 2004). Even vaccines unrelated to intestinal disease may alleviate experimental colitis. Thus, a three-component Bordetella pertussis vaccine attenuated colitis in Gai2deficient mice (Ohman, 2005).

Contrary to the adaptive immunity, the innate immune system would respond immediately (Fellermann et al. 2003). The innate immune system is supposed to play a key role in the protection against IBD through production of defensins and other protective mechanisms (Wehkamp et al. 2003; Wehkamp, 2005; Wehkamp, Schauber and Stange, 2007). However, an important pathogenetic trait of Salmonella is to down-regulate the production of defensins, enabling bacterial invasion of the gut mucosa (Salzman et al. 2003). Experiments on pigs have shown that Salmonella enterica serovar typhimurium infection might up-regulate cathelicidine genes and stimulate the production of another family of antimicrobial peptides prominent in the host defence mechanisms of several mammalian species (Wu et al. 2000). If immunisation with the non-pathogenous salmonella Ty21a has the same effect, it would contribute to protection of the host.

Treating chronic colitis with a vaccine that is well known, very well tolerated and cheap is an attractive idea. Several animal experiments support 
the idea. Unfortunately our results do not. But further studies are warranted before we exclude a positive effect of Ty21a in this context.

\section{References}

Bibiloni, R., Fedorak, R.N., Tannock, G.W., Madsen, K.L., Gionchetti, P., Campieri, M., De Simone, C. and Sartor, R.B. 2005. VSL\#3 probiotic-mixture induces remission in patients with active ulcerative colitis, Am. J. Gastroenterol., 100(7):1539-46.

Boirivant, M., Fuss, I.J., Ferroni, L., De Pascale, M. and Strober, W. 2001. Oral administration of recombinant cholera toxin subunit B inhibits IL-12-mediated murine experimental (trinitrobenzene sulfonic acid) colitis, J. Immunol., 166(5):3522-32.

Carrier, J., Aghdassi, E., Platt, I., Cullen, J. and Allard, J.P. 2001. Effect of oral iron supplementation on oxidative stress and colonic inflammation in rats with induced colitis, Aliment. Pharmacol. Ther., 15(12):1989-99.

Di Giacinto, C., Marinaro, M., Sanchez, M., Strober, W. and Boirivant, M. 2005. Probiotics ameliorate recurrent Th1-mediated murine colitis by inducing IL-10 and IL-10-dependent TGF-beta-bearing regulatory cells, J. Immunol., 174(6):3237-46.

Engels, E.A., Falagas, M.E., Lau, J. and Bennish, M.L. 1998. Typhoid fever vaccines: a meta-analysis of studies on efficacy and toxicity, $B M J$, 316(7125):110-6.

Erichsen, K., Milde, A.M., Arslan, G., Helgeland, L., Gudbrandsen, O.A., Ulvik, R.J., Berge, R.K., Hausken, T. and Berstad, A. 2005. Low-dose oral ferrous fumarate aggravated intestinal inflammation in rats with DSS-induced colitis, Inflamm. Bowel. Dis., 11(8):744-8.

Fellermann, K., Wehkamp, J., Herrlinger, K.R. and Stange, E.F. 2003. Crohn's disease: a defensin deficiency syndrome?, Eur. J. Gastroenterol. Hepatol., 15(6):627-34.

Fonager, K., Sorensen, H.T. and Olsen, J. 1997. Change in incidence of Crohn's disease and ulcerative colitis in Denmark. A study based on the National Registry of Patients, 1981-1992, Int. J. Epidemiol., 26(5):1003-8.

Fujiwara, M., Kaneko, T., Iwana, H., Taketomo, N., Tsunoo, H., Kanno, J., Ohkusa, T. and Okayasu, I. 2003. Inhibitory effects of Bifidobacterium longum on experimental ulcerative colitis induced in mice by synthetic dextran sulfate sodium, Digestion, 67(1-2):90-5.

Holmen, N., Lundgren, A., Lundin, S., Bergin, A.M., Rudin, A., Sjovall, H. and Ohman, L. 2006. Functional CD4+CD25high regulatory T cells are enriched in the colonic mucosa of patients with active ulcerative colitis and increase with disease activity, Inflamm. Bowel. Dis., 12(6):447-56.

Jun, S., Gilmore, W., Callis, G., Rynda, A., Haddad, A. and Pascual, D.W. 2005. A live diarrheal vaccine imprints a Th2 cell bias and acts as an anti-inflammatory vaccine, J. Immunol., 175(10):6733-40.

Kanauchi, O., Mitsuyama, K., Araki, Y. and Andoh, A. 2003. Modification of intestinal flora in the treatment of inflammatory bowel disease, Curr. Pharm. Des, 9(4):333-46.

Kim, H.S. and Berstad, A. 1992, Experimental colitis in animal models, Scand. J. Gastroenterol., 27(7):529-37.

Korzenik, J.R., Dieckgraefe, B.K., Valentine, J.F., Hausman, D.F. and Gilbert, M.J. 2005. Sargramostim for active Crohn's disease, N.Engl. J. Med., 352(21):2193-201.

Kristinson, J., Nygaard, K., Sundseth, A., Aadland, E. and Fagerhol, M.K. 2002, Comparison of faecal and intestinal concentrations of granulocyte marker protein and localization of gastrointestinal tumours in rats, Scandinavian journal of gastroenterology, 37(9):1029-33.

Lapidus, A. 2006, Crohn's disease in Stockholm County during 1990-2001: an epidemiological update, World J. Gastroenterol., 12(1):75-81.
Lavelle, E.C., Jarnicki, A., McNeela, E., Armstrong, M.E., Higgins, S.C., Leavy, O. and Mills, K.H. 2004, "Effects of cholera toxin on innate and adaptive immunity and its application as an immunomodulatory agent", J. Leukoc. Biol., 75(5):756-63.

Lundin, B.S., Johansson, C. and Svennerholm, A.M. 2002. Oral immunization with a Salmonella enterica serovar typhi vaccine induces specific circulating mucosa-homing $\mathrm{CD} 4(+)$ and $\mathrm{CD} 8(+) \mathrm{T}$ cells in humans, Infect. Immun., 70(10):5622-7.

Madsen, K., Cornish, A., Soper, P., McKaigney, C., Jijon, H., Yachimec, C., Doyle, J., Jewell, L. and De Simone, C. 2001. Probiotic bacteria enhance murine and human intestinal epithelial barrier function, Gastroenterology, 121(3):580-91.

Milde, A.M., Arslan, G., Roseth, A., Berstad, A., Overmier, J.B. and Murison, R. 2003. Intestinal permeability and faecal granulocyte marker protein in dextran sulphate sodium - induced colitis in rats, Scandinavian Journal of Laboratory Animal Science, 30(4):169-75.

Milde, A.M. and Murison, R. 2002. A study of the effects of restraint stress on colitis induced by dextran sulphate sodium in singly housed rats, Integr. Physiol Behav. Sci., 37(2):140-50.

Moum, B., Vatn, M.H., Ekbom, A., Aadland, E., Fausa, O., Lygren, I., Stray, N., Sauar, J. and Schulz, T. 1996. Incidence of Crohn's disease in four counties in southeastern Norway, 1990-93. A prospective population-based study. The Inflammatory Bowel South-Eastern Norway (IBSEN) Study Group of Gastroenterologists, Scand. J. Gastroenterol., 31(4):355-61.

Ohman, L. 2005. Acellular Bordetella pertussis vaccine enhances mucosal interleukin-10 production, induces apoptosis of activated Th1 cells and attenuates colitis in Galphai2-deficient mice, Clinical and experimental immunology, 141(1):37-46.

Osman, N., Adawi, D., Ahrne, S., Jeppsson, B. and Molin, G. 2004. Modulation of the effect of dextran sulfate sodium-induced acute colitis by the administration of different probiotic strains of Lactobacillus and Bifidobacterium, Dig. Dis. Sci., 49(2):320-7.

Salzman, N.H., Ghosh, D., Huttner, K.M., Paterson, Y. and Bevins, C.L. 2003. Protection against enteric salmonellosis in transgenic mice expressing a human intestinal defensin, Nature, 422(6931):522-6.

Sands, B.E., Cuffari, C., Katz, J., Kugathasan, S., Onken, J., Vitek, C. and Orenstein, W. 2004. Guidelines for immunizations in patients with inflammatory bowel disease, Inflamm. Bowel Dis., 10(5):677-92.

Uhlig, H.H., Coombes, J., Mottet, C., Izcue, A., Thompson, C., Fanger, A., Tannapfel, A., Fontenot, J.D., Ramsdell, F. and Powrie, F. 2006. Characterization of Foxp3+CD4+CD25+ and IL-10secreting CD4+CD25+ T cells during cure of colitis, J. Immunol., 177( 9):5852-60.

Wehkamp, J. 2005. Reduced Paneth cell alpha-defensins in ileal Crohn's disease, Proceedings of The National Academy of Sciences of The United States of America, 102(50):18129-34.

Wehkamp, J., Harder, J., Weichenthal, M., Mueller, O., Herrlinger, K.R., Fellermann, K., Schroeder, J.M. and Stange, E.F. 2003. Inducible and constitutive beta-defensins are differentially expressed in Crohn's disease and ulcerative colitis, Inflamm. Bowel. Dis., 9(4):215-23.

Wehkamp, J., Schauber, J. and Stange, E.F. 2007. Defensins and cathelicidins in gastrointestinal infections, Curr. Opin. Gastroenterol., 23(1):32-8.

Wu, H., Zhang, G., Minton, J.E., Ross, C.R. and Blecha, F. 2000. Regulation of cathelicidin gene expression: induction by lipopolysaccharide, interleukin-6, retinoic acid, and Salmonella enterica serovar typhimurium infection, Infect. Immun., 68(10):5552-8. 